Revista Brasileira de Agricultura Irrigada v.10, nº.6, p. 1038 - 1048, 2016

ISSN 1982-7679 (On-line)

Fortaleza, CE, INOVAGRI - http://www.inovagri.org.br

DOI: $10.7127 /$ rbai.v10n600497

Protocolo 497.16 - 10/10/2016 Aprovado em 25/11/2016

\title{
ESTIMATIVAS DA EVAPOTRANSPIRAÇÃO DE REFERÊNCIA DO MUNICÍPIO DE ARIQUEMES (RO) UTILIZANDO OS MÉTODOS PENMAN-MONTEITH-FAO E HARGREAVES-SAMANI
}

\author{
Roberto Luís da Silva Carvalho ${ }^{1}$, Angel Ramon Sanchez Delgado ${ }^{2}$
}

\begin{abstract}
RESUMO
O objetivo do estudo foi estimar a evapotranspiração de referência (ETo) do município de Ariquemes (RO) e comparar as estimativas dos métodos Penman-Monteith-FAO e HargreavesSamani, para o período de 2011 a 2013. Foram utilizados os dados de temperatura do ar, umidade relativa, precipitação pluviométrica, velocidade do vendo e radiação solar disponibilizados pelo Instituto Nacional de Meteorologia - INMET e obtidos na estação Meteorológica Automática de Ariquemes/RO. Para comparar as duas metodologias utilizadas foram utilizados o índice de concordância (d), coeficientes de correlação (r), coeficiente de determinação $\left(\mathrm{R}^{2}\right)$ e coeficientes linear e angular da reta de regressão. Dentre os resultados, verificou-se que o Hargreaves-Samani superestima em todos os meses a ETo em relação ao método padrão. No entanto, as estimativas foram consideradas boas.
\end{abstract}

Palavras-chave: uso da água, métodos de estimação, irrigação, solo-planta-atmosfera

\section{ESTIMATING REFERENCE EVAPOTRANSPIRATION OF THE CITY ARIQUEMES (RO) USING THE METHODS PENMAN-MONTEIH-FAO AND HARGREAVES-SAMANI}

\begin{abstract}
The objective of the study was to estimate the reference evapotranspiration (ETo) of the city of Ariquemes (RO) and to compare the estimates by the methods of Penman-Monteith-FAO and Hargreaves-Samani for the period 2011-2013. We used data of air temperature, relative humidity, pluviometer measurements, speed of wind and solar radiation provided by the National Institute of Meteorology - INMET and obtained the Automatic Meteorological Station of Ariquemes/RO. To compare the two methodologies were used the concordance index (d),

\footnotetext{
${ }^{1}$ Professor do Instituto Federal de Educação, Ciência e Tecnologia de Rondônia - Campus Ariquemes e doutorando no Programa de Pós-Graduação em Ciência, Tecnologia e Inovação em Agropecuária na Universidade Federal Rural do Rio de Janeiro - PPGCTIA/UFRRJ, Seropédica- RJ, Brasil. E-mail: roberto.carvalho@ifro.edu.br

2 Professor do Programa de Pós-Graduação em Ciência, Tecnologia e Inovação em Agropecuária (PPGCTIA/UFRRJ), Instituto de Ciências Exatas, Seropédica-RJ, Brasil. E-mail: asanchez@ufrrj.br
} 
the correlation coefficients (r), the coefficient of determination $\left(\mathrm{R}^{2}\right)$ and linear and angular coefficients of the regression line. Among the results, we found that the Hargreaves-Samani overestimates in every month the ETo when compared to standard method. However, the estimates were considered good.

Keywords: water use, estimation methods, irrigation, soil-plant-atmosphere

\section{INTRODUÇÃO}

Uma variável de suma importância para os produtores que utilizam a irrigação é a "evapotranspiração de uma cultura agrícola” (ETc), sendo esta necessária para a otimização do uso da água na irrigação (PEIXOTO et al., 2010). No entanto, tal variável é considerada de difícil obtenção, sendo assim se utiliza a evapotranspiração de referência (ETo) (DOORENBOS; PRUITT, 1977) juntamente com o coeficiente da cultura (Kc) para dimensionar o uso da água (ALVES SOBRINHO et al., 2011; CARVALHO et al., 2011).

A evapotranspiração (ET) é definida como um processo combinado de transferência de água para a atmosfera por evaporação da água do solo e por transpiração das plantas (DOORENBOS; PRUITT, 1977; MENDONÇA et al. 2003; TESTEZLAF, 2011), no qual esse processo pode ser desmembrado nas suas componentes de transpiração, evaporação de água interceptada, evaporação do solo e a evaporação de superfícies livres de água.

A evapotranspiração de referência é definida como a evapotranspiração de uma cultura hipotética (normalmente grama) que cobre todo o solo, em crescimento ativo, sem restrição hídrica e nutricional, com parâmetros de altura entre $0,08 \mathrm{~m}$ a $0,15 \mathrm{~m}$, albedo igual a 0,23 e resistência da cultura ao transporte de vapor d'água igual a $70 \mathrm{~s} \mathrm{~m}^{-1}$ (ALLEN et al., 1998; CARVALHO et al., 2011).

O coeficiente da cultura (Kc) é um parâmetro relacionado aos fatores ambientais e fisiológicos das plantas, que geralmente são ajustados para as condições locais nos quais será utilizado, e variam de acordo com a fase do desenvolvimento da cultura (DOORENBOS; KASSAM, 1994).

A mensuração da evapotranspiração se refere ao total da perda de água para a atmosfera, da superfície do solo e das plantas. Sendo assim, o valor numérico de água evapotranspirada num sistema solo-planta-atmosfera, torna-se uma informação fundamental no planejamento, dimensionamento e manejo de cultivos irrigados. Diante disso, pode-se determinar corretamente a quantidade e o momento da aplicação de água pela irrigação, maximizando assim o uso dos recursos hídricos da região a nível econômico e ambiental (CAVALCANTE et al., 2011).

Vários métodos de estimativa da evapotranspiração de referência têm sido desenvolvidos e testados visando à adequação do método às condições climáticas de uma dada região, pela simplificação de uso ou pela falta de elementos climáticos que alimentem os métodos (HARGREAVES; ALLEN, 2003; CARVALHO et al., 2011).

Os métodos variam de uma abordagem simples, que requerem apenas dados de temperatura, até abordagens mais complexas baseadas em modelos físicos, que requerem uma série de dados de entrada, por exemplo o modelo Penman-Monteith, (ALLEN et al., 1998), que é utilizado como padrão da FAO - Food and Agriculture Organization.

$\mathrm{Na}$ atualidade, tais métodos podem ser classificados em dois grandes grupos: o primeiro grupo inclui os métodos onde a evapotranspiração pode ser determinada a partir de medidas diretas como os lisímetros, balanço hídrico e controle de umidade no solo. No segundo grupo estão os métodos determinados através de dados climáticos, também chamados de métodos indiretos, nos quais a evapotranspiração de referência é avaliada por fórmulas empíricas e racionais ou modelos físico-matemáticos.

Segundo Oliveira et al. (2001) é de grande importância que estas fórmulas sejam testadas antes de serem utilizadas em locais onde o clima não seja semelhante ao clima de onde o método 
foi elaborado. Por outro lado, a escolha do método de estimação deve levar em consideração tanto a praticidade, bem como a precisão, posto que sua utilização na prática diária por parte do produtor deva ser a mais simplificada possível.

Além disso, Scaloppi e Garcia (2015) afirmam que a estimação da demanda diária pode, em períodos típicos semanais, quinzenais ou mensais, pode ser ainda mais simplificada, visto que as condições micrometeorológicas e/ou a intensidade da radiação atmosférica de determinadas regiões possam vir a não sofrerem modificações significativas, sugerindo assim a utilização de processos cada vez mais simples visando estimular aos produtores sua utilização no dimensionamento quanto do manejo de irrigações.

O método padrão FAO (PenmanMonteith) necessita de uma gama de variáveis nem sempre disponíveis em algumas localidades, sendo necessária a utilização de outros métodos mais simples em seu cálculo.
Dentre esses métodos mais simples, reaplicáveis e que utilizam poucos dados meteorológicos, tem-se o método de Hargreaves-Samani (1982, 1985) que utiliza em sua equação dados de temperatura máxima e mínima do ar. Sendo assim, visando conhecer o comportamento da evapotranspiração de referência em Ariquemes RO, o objetivo do presente estudo é estimar a evapotranspiração de referência do município e comparar as estimativas pelos métodos PenmanMonteith e Hargreaves-Samani, para o período de 2011 a 2013.

\section{MATERIAL E MÉTODOS}

O município de Ariquemes localizase na região tropical, próximo a linha do Equador, entre as latitudes $09^{\circ} 41^{\prime} \mathrm{S}$ e $10^{\circ}$ $18^{\prime} \mathrm{S}$ e longitudes de $62^{\circ} 24^{\prime} \mathrm{W}$ e $63^{\circ} 37^{\prime} \mathrm{W}$. Situa-se na mesorregião do Leste Rondoniense - Microrregião de Ariquemes (Figura 1).

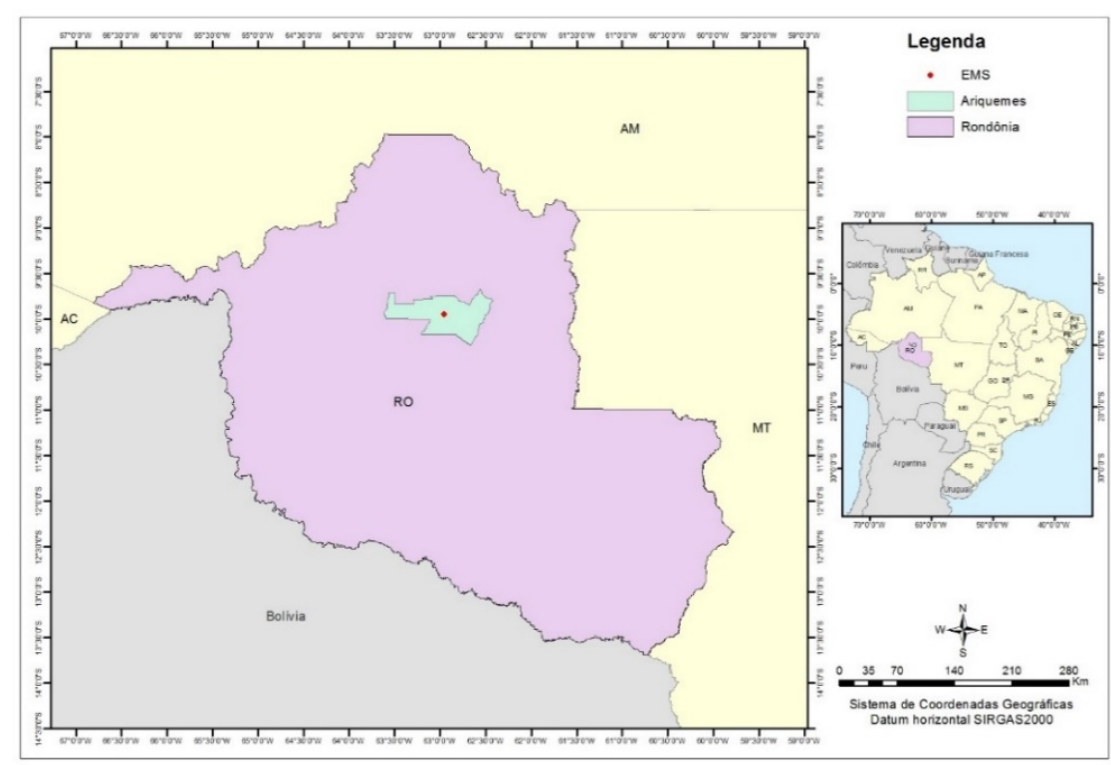

Figura 1. Mapa da localização da estação meteorológica automática de Ariquemes.

O clima de Ariquemes, segundo a classificação de KÖPPEN, é do tipo Aw - Clima Tropical Chuvoso com media climatológica de temperatura do ar de $25,5^{\circ} \mathrm{C}$, sendo praticamente constante no decorrer do ano e apresentando uma amplitude térmica mensal em torno de $10^{\circ} \mathrm{C}$. A temperatura máxima em média é de $32,1^{\circ} \mathrm{C}$, enquanto a temperatura mínima em média é superior a $18^{\circ} \mathrm{C}$ durante todo o ano, com exceção de alguns dias durante o período de inverno quando ocorre o fenômeno denominado de "friagem", quando a temperatura chega a $15^{\circ} \mathrm{C}$. As maiores amplitudes térmicas anuais ocorrem, geralmente, nos meses de julho e agosto, sendo esta uma característica do clima Aw. A precipitação total anual é em torno de 
$2290 \mathrm{~mm} \mathrm{ano}^{-1}$. A região possui duas estações bem definidas sendo uma estação chuvosa com sete meses de duração, com ocorrência de outubro a abril e a estação seca, de junho a agosto, onde as chuvas são escassas. Os meses de maio e setembro são considerados meses de transição entre regimes. A região é caracterizada como de clima úmido, com média anual da umidade relativa em torno de $82 \%$ podendo atingir valores médios abaixo dos $70 \%$ durante os meses de seca (CARVALHO et al., 2016).

O município possui uma área territorial de 4.426,6 $\mathrm{Km}^{2}$. Segundo projeções do IBGE, em 2016 sua população está estimada em 105.896 habitantes (IBGE, 2016). Sua economia é baseada principalmente (1) na agricultura (milho, café, feijão e soja), (2) na pecuária de corte e leite e (3) na piscicultura, setor este em grande expansão e tem, atualmente, desenvolvido a economia local (CARVALHO et al., 2016).

Foram utilizados os dados de temperatura do ar, umidade relativa, precipitação pluviométrica, velocidade do vendo e radiação solar disponibilizados pelo Instituto Nacional de
Meteorologia - INMET. As variáveis foram obtidas na estação Meteorológica Automática de Ariquemes/RO (Figura 1) (09 $56^{\prime} \mathrm{S}$ e $62^{\circ} 57^{\prime} \mathrm{W}$, $140 \mathrm{~m})$, no período de janeiro de 2011 até fevereiro de 2013.

Foram utilizados dois métodos de estimação da evapotranspiração, o HargreavesSamani (HARGREAVES; SAMANI, 1982) e o método padrão da FAO Penman-Monteith (ALLEN et al., 1998). Apesar do método Hargreaves-Samani ser idealizado para regiões com clima de regiões semi-áridas, optou-se por ele, principalmente, para estimar com maior eficiência a ETo nos meses de seca, onde ocorre uma grande mudança no padrão climatológico da região, com umidades inferiores a $70 \%$ e temperaturas mais elevadas. Por outro lado, este método é de simples de aplicação, podendo assim ser utilizado por produtores locais. A escolha do método padrão FAO se deu por ser um método aplicável a todas as regiões.

O método Hargreaves-Samani requer os dados de temperatura, pois se baseia na temperatura média do ar e na amplitude térmica. Seguindo a seguinte formulação:

$$
\mathrm{ET}_{\mathrm{o}}=0,0135 \mathrm{~K}_{\mathrm{T}}\left(\mathrm{T}_{\text {Med }}+17,8\right)\left(\mathrm{T}_{\mathrm{Max}}-\mathrm{T}_{\text {Min }}\right)^{0,5} \mathrm{R}_{\mathrm{a}}
$$

Como normalmente substitui-se a parcela $0,0135 K_{T}$ por 0,0023 , pois $K_{T}=0,17$ para

regiões de interior e $K_{T}=0,2$ para regiões costeiras. Temos:

$$
\mathrm{ET}_{\mathrm{o}}=0,0023\left(\mathrm{~T}_{\text {Med }}+17,8\right)\left(\mathrm{T}_{\text {Max }}-\mathrm{T}_{\text {Min }}\right)^{0,5} \mathrm{R}_{\mathrm{a}}
$$

Onde (Ra) é a radiação solar no topo da atmosfera, em $\mathrm{MJ} \mathrm{m}^{-2} \mathrm{~d}^{-1}$, definida por:

$$
R a=\frac{118,08}{\pi} d_{r}\left[\omega_{s} \operatorname{sen}(\varphi) \operatorname{sen}(\delta)+\cos (\varphi) \cos (\delta) \operatorname{sen}\left(\omega_{s}\right)\right]
$$

Tem-se que $\varphi$ é o valor da latitude local em radianos e $d_{r}$ é a distância relativa entre a terra e o sol (rad) obtida por:

$$
d_{r}=1+0,033 \cos \left(\frac{2 \pi}{365} J\right)
$$

com j o dia do ano. A declinação solar $\delta$ foi calculada por :

$$
\delta=0,409 \operatorname{sen}\left(\frac{2 \pi}{365} J-1,39\right)
$$

O ângulo horário ao nascer do sol $\left(\omega_{s}\right)$ foi calculado pela expressão:

$$
\omega_{s}=\arccos [-\tan (\varphi) \tan (\delta)]
$$

O método da FAO (Penman-Monteith) é sintetizado na seguinte equação (ALLEN et al. 1998):

$$
\text { ETo }=\frac{0,408 \Delta\left(\mathrm{R}_{\mathrm{n}}-\mathrm{G}\right)+\gamma \frac{900}{\mathrm{~T}+273} \mathrm{U}_{2}\left(\mathrm{e}_{\mathrm{s}}-\mathrm{e}_{\mathrm{a}}\right)}{\Delta+\gamma\left(1+0,34 \mathrm{U}_{2}\right)} .
$$

Onde: 
ETo - evapotranspiração de referência $\left(\mathrm{mm} \mathrm{d}^{-1}\right)$;

$\Delta$ - declividade da curva de pressão de vapor na saturação versus temperatura do ar $\left(\mathrm{KPa}{ }^{0} \mathrm{C}^{-1}\right)$;

$\mathrm{R}_{\mathrm{n}}$ - saldo de radiação na superfície do cultivo (MJ m ${ }^{-2} \mathrm{~d}^{-1}$ );

$G$ - densidade de fluxo de calor no solo $\left(\mathrm{MJ} \mathrm{m}^{-2} \mathrm{~d}^{-1}\right)$, sendo $(\mathrm{G}=0)$ para dados diários;

$\gamma$ - constante psicrométrica $\left(\mathrm{KPa}^{0} \mathrm{C}^{-1}\right)$;

$\mathrm{U}_{2}$ - velocidade do vento a $2 \mathrm{~m}$ de altura $\left(\mathrm{m} \mathrm{s}^{-1}\right)$;

$\mathrm{e}_{\mathrm{s}}$ - pressão de vapor na saturação $\mathrm{KPa}{ }^{0}$;

$\mathrm{e}_{\mathrm{a}}$ - pressão de vapor atual, $\mathrm{KPa}{ }^{0}$;

$\mathrm{T}$ - temperatura média do ar tomada a $2 \mathrm{~m}$ de altura $\left({ }^{\circ} \mathrm{C}\right)$.

$\frac{T_{\operatorname{Max}}+T_{\text {Min }}}{2}$

O cálculo de $\mathrm{T}$ é defino por: $T=$

A declividade da curva de saturação $\Delta$ foi calculada pela seguinte equação:

$$
\Delta=\frac{4098\left[0,6108 \exp \left(\frac{17,27 T}{T+237,3}\right)\right]}{(T+237,3)^{2}} \text {. }
$$

A constante psicrométrica $\gamma$ foi calculada pela expressão:

$$
\gamma=0,665 \times 10^{-3} \text { Patm. }
$$

Por sua vez a pressão atmosférica local (Patm) foi calculada com base na altitude local (z), em metros:

$$
\text { Patm }=101,3\left(\frac{293-0,0065 z}{293}\right)^{5,26} .
$$

A saturação da pressão de vapor de uma determinada temperatura t é calculada por

$$
\mathrm{e}^{0}(\mathrm{t})=0,6108 \exp \left[\frac{17,27 t}{t+237,3}\right] \text {. }
$$

Com isso, para se encontrar os valores da pressão de vapor saturação $\left(e_{s}\right)$ e pressão de vapor atual $\left(e_{a}\right)$ foram utilizadas as expressões:

$$
\begin{aligned}
& e_{s}=\frac{\mathrm{e}^{0}\left(T_{\max }\right)+\mathrm{e}^{0}\left(T_{\min }\right)}{2} \mathrm{e} \\
& e_{a}=\frac{\mathrm{e}^{0}\left(T_{\min }\right) \frac{U R_{\max }}{100}+\mathrm{e}^{0}\left(T_{\max }\right) \frac{U R_{\min }}{100}}{2},
\end{aligned}
$$

em que UR é a umidade relativa do ar (\%).

O déficit de saturação foi calculado pela diferença destas expressões $\left(e_{s}-e_{a}\right)$.

A estimativa do saldo de radiação $(\mathrm{Rn})$ foi obtida pela expressão:

$$
R n=R n s-R n l .
$$

$R n s$ é o saldo da radiação de ondas curtas $\left(\mathrm{MJ} \mathrm{m}^{-2} \mathrm{~d}^{-1}\right)$, obtido por $\mathrm{Rns}=(1-\alpha) \mathrm{Rs}$, com o valor de $\alpha=0,23$ referente ao coeficiente de reflexão da vegetação (albedo) e Rs é radiação solar incidente $\left(\mathrm{MJ} \mathrm{m}^{-2} \mathrm{~d}^{-1}\right)$, mensurada na estação meteorológica.

$R n l$ é o saldo de radiação de ondas longas, calculada por:

$$
R n l=\sigma\left[\frac{\left(T_{\max }+273,16\right)^{4}+\left(T_{\min }+273,16\right)^{4}}{2}\right]\left(0,34-0,14 \sqrt{e_{a}}\right)\left(1,35 \frac{R s}{R s o}-0,35\right) .
$$

Em que:

$\sigma$ é a constante de Stefan-Boltzmann $(4,903 \times$ $\left.10^{-9} \mathrm{MJ} \mathrm{m}^{-2} \mathrm{~d}^{-1}\right) ; \quad T_{\max }$ e $T_{\min }$ são as temperaturas máximas e mínimas, em ${ }^{\circ} \mathrm{C}$, do dia, respectivamente.

A radiação solar incidente na ausência de nuvens (Rso) foi calculada por:

$$
\text { Rso }=\left(0,75+2 \times 10^{-5} z\right) R a \text {. }
$$

Para comparação dos métodos foram utilizadas as seguintes medidas: índice de concordância (d) e coeficiente de confiança (c) (WILLMOTT et al., 1985; CAMARGO; SENTELHAS, 1997), coeficientes de correlação (r), coeficiente de determinação $\left(\mathrm{R}^{2}\right)$ e os coeficientes linear e angular da reta de regressão linear (MONTGOMERY; RUNGER, 2009). Para comparação das médias mensais das estimativas foi utilizado o teste $t$ de Student para 
dados pareados (MONTGOMERY; RUNGER, 2009).

Segundo Willmott et al. (1985) o índice de concordância (d) expressa a aproximação dos valores de ETo estimados pelos métodos Hargreaves-Samani e Penman-Monteith, que é o método padrão da FAO. Sendo assim o índice foi calculado por:

$$
\mathrm{d}=1-\left[\frac{\sum\left(\mathrm{P}_{\mathrm{i}}-\mathrm{O}_{\mathrm{i}}\right)^{2}}{\sum\left(\left|\mathrm{P}_{\mathrm{i}}-\overline{\mathrm{O}}\right|+\left|\mathrm{O}_{\mathrm{i}}-\overline{\mathrm{O}}\right|\right)^{2}}\right]
$$

Onde:

$P_{i}$ - valor estimado por HargreavesSamani e suas variações $\left(\mathrm{mm} \mathrm{d}^{-1}\right)$;

$\mathrm{O}_{\mathrm{i}}$ - valor calculado pelo método de Penman-Monteih $\left(\mathrm{mm} \mathrm{d}^{-1}\right)$;

$\overline{0}$ - média dos valores calculada pelo método de Penman-Monteih.

O coeficiente de correlação de Pearson (r) foi calculado pela seguinte equação:

$$
r=\frac{\sum_{\mathrm{i}=1}^{\mathrm{n}} \mathrm{p}_{\mathrm{i}} \mathrm{o}_{\mathrm{i}}-\mathrm{n} \overline{\mathbf{p}} \overline{\mathbf{o}}}{\sqrt{\left(\sum_{\mathrm{i}=1}^{\mathrm{n}} \mathrm{p}_{\mathrm{i}}^{2}-\mathrm{n} \overline{\mathrm{p}}^{2}\right)\left(\sum_{\mathrm{i}=1}^{\mathrm{n}} \mathrm{o}_{\mathrm{i}}^{2}-\mathrm{no}^{2}\right)}}
$$

Este coeficiente varia no intervalo $[-1,1]$, isto é, $-1 \leq \mathrm{r} \leq 1$. Onde $\overline{\mathrm{p}}$ é a média dos valores estimados por Hargreaves-Samani. No entanto, espera-se que a correlação entre as estimativas sejam sempre positivas; ou seja, $0 \leq$ $r \leq 1$. O coeficiente de correlação $(r)$ pode ser classificado (COHEN, 1988) como: "muito baixo" ( $\mathrm{r}<0,1)$; "baixo" $(0,1<\mathrm{r}<0,3)$; "moderado" $(0,3<\mathrm{r}<0,5)$; “alto" $(0,5<\mathrm{r}<0,7)$; "muito alto" $(0,7<\mathrm{r}<0,9)$; e “quase perfeito" ( $\mathrm{r}$ $>0,9$ ). O coeficiente de concordância (c) ou de ajuste de Willmott é dado por:

$$
\mathrm{c}=\mathrm{r} \times \mathrm{d}
$$

Este índice mede o desempenho de acordo com as seguintes classes sugeridas por Camargo e Sentelhas (1997): péssimo, c $\leq 0,40 ;$ mau, 0,41 $\leq \mathrm{c} \leq 0,50$; sofrível, $0,51 \leq \mathrm{c} \leq 0,60$; mediano, $0,61 \leq \mathrm{c} \leq 0,65$; bom, $0,66 \leq \mathrm{c} \leq 0,75$; muito bom, $0,76 \leq \mathrm{c} \leq 0,85$; e ótimo, $\mathrm{d}>0,85$.

Os coeficientes angular $\left(b_{1}\right)$ e linear $\left(b_{0}\right)$ da regressão linear foram calculados pelas equações 19 e 20.

$$
\begin{aligned}
\mathrm{b}_{1} & =\frac{\sum \mathrm{p}_{\mathrm{i}} \mathrm{o}_{\mathrm{i}}-\frac{1}{\mathrm{n}}\left(\sum \mathrm{p}_{\mathrm{i}}\right)\left(\sum \mathrm{o}_{\mathrm{i}}\right)}{\sum \mathrm{p}_{\mathrm{i}}^{2}-\frac{1}{\mathrm{n}}\left(\sum \mathrm{p}_{\mathrm{i}}\right)^{2}} \\
\mathrm{~b}_{0} & =\overline{\mathrm{o}}-\mathrm{b}_{1} \overline{\mathrm{p}}
\end{aligned}
$$

O coeficiente de determinação $\left(\mathrm{R}^{2}\right)$, que indica quantos por cento a variação explicada pela regressão representa da variação total, será calculado através da equação 8:

$$
\mathrm{R}^{2}=\frac{\mathrm{b}_{1} \sum \mathrm{p}_{\mathrm{i}} \mathrm{o}_{\mathrm{i}}-\frac{1}{\mathrm{n}}\left(\sum \mathrm{p}_{\mathrm{i}}\right)\left(\sum \mathrm{o}_{\mathrm{i}}\right)}{\sum \mathrm{o}_{\mathrm{i}}^{2}-\frac{1}{\mathrm{n}}\left(\sum \mathrm{o}_{\mathrm{i}}\right)^{2}} \times 100
$$

O coeficiente de determinação varia entre 0 e 1 ; isto é, $0 \leq \mathrm{R}^{2} \leq 1$.

A estatística do teste $t$ de Student para dados pareados foi calculada da seguinte forma:

$$
T_{0}=\frac{\bar{D}-\Delta_{0}}{S_{D} / \sqrt{n}} .
$$

Com a diferença entre cada par de observações definida por $D_{j}=X_{1 j}-X_{2 j}$ e $j=$ $1,2,3, \ldots, n$. $\bar{D}$ é a média amostral das $n$ diferenças $D_{1}, D_{2}, D_{3}, \ldots, D_{n}$ e $S_{D}$ é o desvio padrão amostral destas diferenças.

\section{RESULTADOS E DISCUSSÃO}

O índice de concordância observado entre os métodos foi de $d=0,849$ e foi observada uma correlação forte positiva ( $\mathrm{r}=$ $0,818 ; p=0,000)$ entre as variáveis, sendo classificada como "muito alta", nas faixas descritas por Cohen (1988). O índice de confiança encontrado foi de $c=0,695$, sendo este considerado como "bom”, de acordo com as faixas classificação de Camargo e Sentelhas (1997).

A reta de regressão linear melhor ajustada $(F=2208,29 ; p=0,000)$ apresentou os seguintes coeficientes: $b_{1}=1,028(p=$ $0,000)$ e $b_{0}=-0,503(p=0,000) . \quad O$ coeficiente de determinação da reta de regressão linear obtido foi de $R^{2}=0,669$. Na Figura 2 são apresentadas as distribuições dos métodos de estimação. 


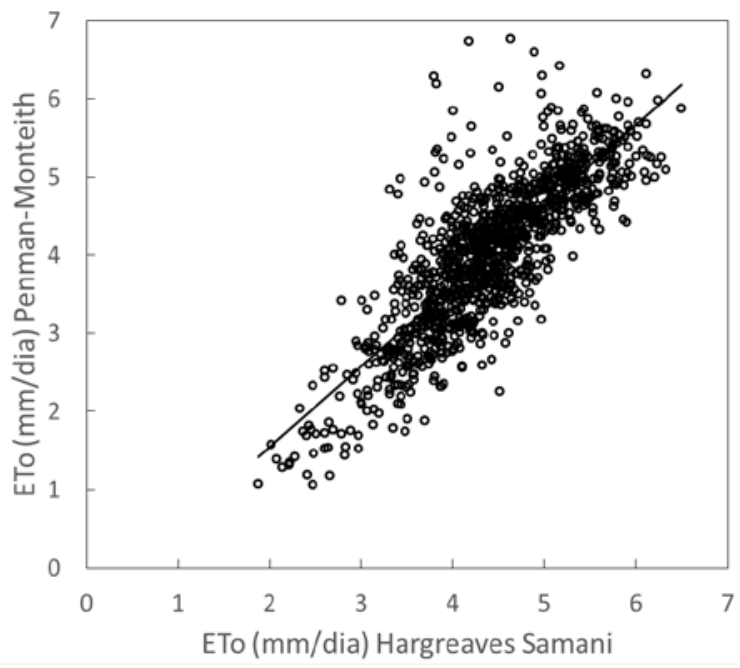

$y=1,028 x-0,503$

$R^{2}=0,6687$

Figura 2. Diagrama de dispersão das estimativas dos métodos Penman-Monteith e Hargreaves-Samani. Fonte: INMET (2011- 2013).

Os valores médios mensais encontrados para as estimativas (ETo) estão descritos na Tabela 1. Observou-se que as médias mensais das estimativas do Método Hargreaves-Samani são maiores que as médias mensais do método Penman-Monteith, ao nível de significância de 0,05 , pelo teste t de Student para dados pareados. Ou seja, o teste Hargreaves-Samani superestimou os valores de ETo. Sanches et al. (2015) encontraram também superestimativas com esse método para a região Norte do Brasil.

Tabela 1 - Comparação das médias mensais das estimativas de ETo (mm/dia) dos métodos PenmanMonteith e Hargreaves-Samani, no período de 2011-2013, em Ariquemes (RO).

\begin{tabular}{|c|c|c|c|c|c|c|c|c|c|c|c|}
\hline \multirow[t]{2}{*}{ Mês } & \multicolumn{4}{|c|}{ Penman-Monteith } & \multicolumn{4}{|c|}{ Hargreaves-Samani } & \multirow[t]{2}{*}{ Diferença } & & \multirow[t]{2}{*}{$\mathrm{p}$} \\
\hline & $\overline{\bar{x}}$ & Máx. & Mín. & $\mathrm{S}$ & $\bar{x}$ & Máx. & Mín. & $\mathrm{S}$ & & & \\
\hline AN & 3,50 & 5,41 & 1,87 & 1,01 & 4,10 & 5,82 & 1,08 & 0,70 & & $-12,168$ & \\
\hline $\mathrm{EV}$ & 47 & 5,62 & 2,27 & $1,($ & 4,06 & 5,38 & 1,06 & & & & \\
\hline IAR & 61 & 5,2 & 08 & & 17 & & 1,28 & & & & \\
\hline $\mathrm{BR}$ & 3,85 & 4,94 & 2,33 & 0,75 & 3,95 & 5,01 & 1,18 & 0 , & -0 & & 0,017 \\
\hline AI & 72 & 5,85 & 2,22 & $0, \epsilon$ & 3,81 & 5,47 & 1,34 & & $-0,09$ & $-2,565$ & 0,012 \\
\hline $\mathrm{N}$ & & 4,7 & 37 & & & & 1,7 & & & & \\
\hline JUL & 32 & 5,31 & 2,70 & 0,62 & 4,45 & 5,85 & 2,00 & & $-0,13$ & 598 & 0,011 \\
\hline AGO & 4,99 & 6,50 & 3,43 & 0,72 & 5,26 & 6,76 & 2,25 & & & -2, & 0,004 \\
\hline $\mathrm{ET}$ & 4,82 & 6,33 & 3,42 & & 5,32 & 6,00 & 3,36 & & -0 & $-9,5$ & 0,000 \\
\hline OUT & & 6,24 & 3,28 & & 480 & 6,08 & 2,30 & & $-0,55$ & & 0,000 \\
\hline NOV & 4,00 & 6,11 & 2,41 & 0,95 & 4,50 & 6,32 & 1,69 & & & $-10,434$ & 0,000 \\
\hline DEZ & 3,88 & 5,81 & 2,61 & 1,03 & 4,39 & 5,86 & 1,52 & 0,68 & $-0,51$ & $-9,864$ & 0,000 \\
\hline
\end{tabular}

Da mesma forma, observa-se na Tabela 1 que durante o período de abril a agosto, os valores estimados para Hargreaves-Samani sobrestimaram a ETo entre 0,10 a $0,27 \mathrm{~mm} /$ dia, em média, enquanto que para os demais meses os valores foram sobrestimados em cerca de 0,50 mm/dia, em média. No mês de agosto foram observadas as maiores máximas estimadas em ambos os métodos.

No estudo realizado por Pereira et al. (2009) objetivando estimar a evapotranspiração de referência durante os períodos chuvoso (outubro a março) e seco (abril a setembro), para a bacia hidrográfica do ribeirão Lavrinha, região da Serra da Mantiqueira, Minas Gerais (MG), 
nos anos de 2007 e 2008, afirmaram que as estimativas do método Hargreaves-Samani foram consideradas "sofríveis" quando comparados ao método padrão. Alencar et al. (2011) afirmaram que para as condições climáticas dos municípios de Montes Claros, Espinosa e Salinas, da região Norte de Minas Gerais, o método de Hargreaves-Samani tende a superestimar, sendo considerado como pior desempenho para as condições climáticas daqueles locais. No entanto, autores citaram que este método foi desenvolvido para a condição semiárida do Nordeste brasileiro, com isso seu desempenho melhorou nos meses mais secos (outubro a março). Mendoza et al. (2016) classificaram as estimativas do método Hargreaves-Samani como "medianas" para a região de São Luís, MA, mas ressaltaram que dentre os métodos que se utilizam dos dados de temperatura esse obteve o melhor desempenho c $=0,64$. Palaretti et al. (2014) em seu estudo na cidade de Bebedouro - SP, com altitude de 601m, com clima tipo Aw, segundo a classificação climática de Köppen modificada, observaram que o método Hargreaves - Samani superestimava valores mensais de ETo em 13\% comparado ao método padrão.
Por outro lado, Tagliaferre et al. (2012) buscando avaliar o desempenho dos métodos na estimativa da ETo para os municípios de Anagé, Piatã e Ilhéus, na Bahia, nos anos de 2006 e 2007, verificaram que Hargreaves-Samani subestimou os valores de ETo, sendo considerado, ainda, como medianas suas estimativas.

Magalhães e Cunha (2012) encontraram para diferentes localidades de Mato Grosso do Sul resultados satisfatórios na comparação dos métodos. Os estudos citados reforçam a necessidade de se buscar o método mais adequado para determinada região. Segundo Lacerda e Turco (2015), os resultados podem variar completamente devido às características climáticas das regiões.

Apesar das médias do Hargreaves-Samani serem maiores para Ariquemes, quando se avaliam as distribuições dos dois métodos (Figura 3), se observa que em algumas estimativas os valores máximos do PenmanMonteith são maiores que os valores da estimativa Hargreaves-Samani, com isso percebe-se que este último método apresentar menor amplitude.

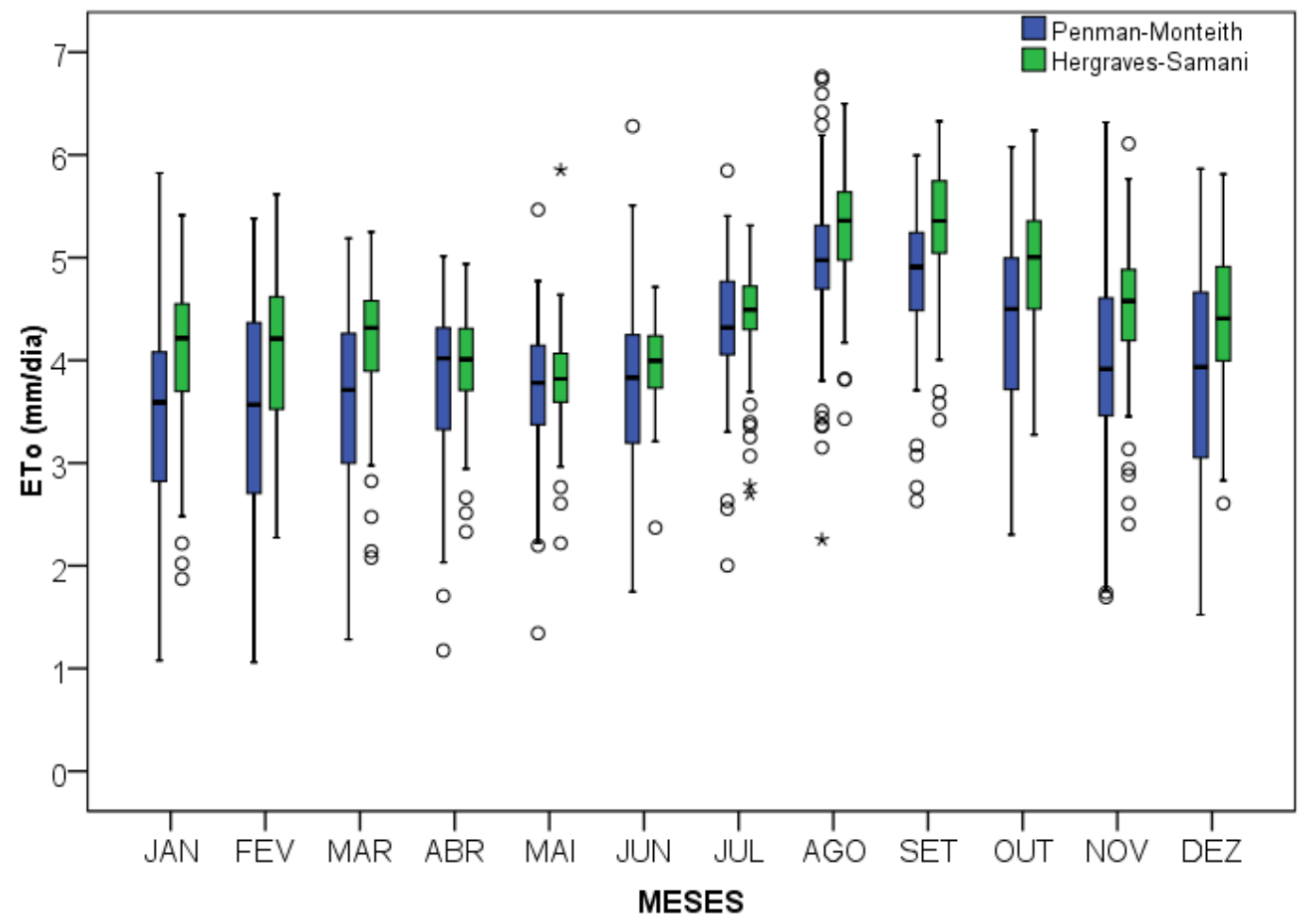

Figura 3. Distribuições das estimativas mensais da ETo pelos métodos Penman-Monteith e Hargreaves-Samani. Fonte: INMET (2011 a 2013). 


\section{CONSIDERAÇÕES FINAIS}

O presente estudo contribuiu para identificar o comportamento da evapotranspiração de referências do município de Ariquemes (RO) no período de 2011-2013, sendo esta uma variável de suma importância para os produtores que utilizam a prática de irrigação. Verificou-se que o HargreavesSamani superestimou em todos os meses a ETo em relação ao método padrão. No entanto, as estimativas foram consideradas boas.

Futuros estudos são sugeridos com outros métodos de estimação que venham a se adequar melhor ao clima de Ariquemes. Da mesma forma, propõe-se o estudo de fatores de correção mensais para as estimativas do HargrevesSamani, visto que em média superestima os valores.

\section{AGRADECIMENTOS}

À Fundação de Amparo ao Desenvolvimento das Ações Científicas e Tecnológicas e a Pesquisa de Rondônia FAPERO, pelo auxílio financeiro através do Programa de bolsas de mestrado e doutorado para docentes (MS / DR / DOCENTE / FAPERO / CAPES - 07/2014).

\section{REFERÊNCIAS}

ALLEN, R. G.; PEREIRA, L. S.; RAES, D.; SMITH, M. Crop evapotranspiration: guidelines for computing crop water requirements. Rome: FAO, 1998. 300 p. (Irrigation and Drainage Paper, 56).

ALENCAR, L. P.; SEDIYAMA, G. C.; WANDERLEY, H. S.; ALMEIDA, T. S.; DELGADO, R. C. Avaliação de métodos de estimativa da evapotranspiração de referência para três localidades no norte de Minas Gerais. Engenharia na agricultura, v.19, n.5, p. 437449, 2011.

ALVES SOBRINHO, T.; RODRIGUES, D. B. B.; OLIVEIRA, P. T. S.; REBUCCI, L. C. S.;
PERTUSSATTI, C. A. Estimativa da evapotranspiração de referência através de redes neurais artificiais. Revista Brasileira de Meteorologia, v.26, n.2, p.197-203, 2011.

CAMARGO, A. P.; SENTELHAS, P. C. Avaliação do desempenho de diferentes métodos de estimativa da evapotranspiração potencial no Estado de São Paulo, Brasil. Revista Brasileira de Agrometeorologia, Santa Maria, v. 5, n. 1, p.89-97, 1997.

CARVALHO, L. G.; RIOS, G. F. A.; MIRANDA, W. L.; CASTRO NETO, P. Evapotranspiração de referência: uma abordagem atual de diferentes métodos de estimativa. Pesquisa

Agropecuária Tropical , v. 41, n. 3, p. 456-465, 2011.

CARVALHO, R. L. S.; NASCIMENTO, B. I. S.; QUERINO, C. A. S.; SILVA, M. J. G.; DELGADO, A. R. S. Comportamento das séries temporais de temperatura do ar, umidade e precipitação pluviométrica no município de Ariquemes (Rondônia-Brasil). Revista Brasileira de Climatologia, n. 12, v. 18, p.123-142, 2016.

CAVALCANTE, J. A. C.; DELGADO, A. R. S.; MEDEIRO, J. A. C. C.; CARVALHO, D. Usos de recursos na dose certa: Uma ferramenta computacional para otimização agrícola. Revista Eletrônica Sistemas \& Gestão, v.6, n.4, p. 398-413, 2011.

COHEN, J. Statistical power analysis for the behavioral sciences. New Jersey: Lawrence Erlbaum, 1988, 569 p.

DOORENBOS, J.; KASSAM, A. H. Efeito da água no rendimento das culturas. Campina Grande: UFPB, 1994. 306p. Estudos FAO: Irrigação e Drenagem, 33.

DOORENBOS, J.; PRUITT, W. O. Guidelines for predicting crop water requirements. Rome: FAO, 179p. 1977. (FAO: Irrigation and Drainage Paper, 24). 
HARGREAVES, G. H., SAMANI, S. Estimating potential evapotranspiration. Journal of Irrigation Drainage Division, v. 108, n. IR3, p. 223-230, 1982.

HARGREAVES, G. H.; SAMANI, Z. A., Reference crop evapotranspiration from temperature. Applied Engineering in Agriculture, v.1, n.2, p.96-99, 1985.

HARGREAVES, G. H.; ALLEN, R. G. History and Evaluation of Hargreaves Evapotranspiration Equation. Journal of Irrigation and Drainage Engineering, v.129, n.1, p.53-63, 2003.

INSTITUTO BRASILEIRO DE GEOGRAFIA E ESTATÍTICA. Levantamento Sistemático da Produção Agrícola (LSPA): Projeções. 2014. Disponível em $<$ http://www.sidra.ibge.gov.br/bda/prevsaf/defa ult.asp?t=1\&z=t\&o=26\&u2=1\&u3=1\&u4=1\&u $1=11>$. Acesso em 24/09/2014.

INSTITUTO BRASILEIRO DE GEOGRAFIA E ESTATÍTICA. Pesquisa Pecuária Municipal. 2014. Disponível em $<$ http://www.sidra.ibge.gov.br/bda/tabela/prota bl.asp? $c=3940 \& z=p \& o=27 \& i=P>$. Acesso em 14/08/2015.

INSTITUTO BRASILEIRO DE GEOGRAFIA E ESTATÍSTICA. Diretoria de Pesquisas, Coordenação de População e Indicadores Sociais. Estimativas da população residente no Brasil e unidades da federação com data de referência em $\mathbf{1}^{\mathbf{0}}$ de julho de 2016. 2016. Disponível em $<\mathrm{ftp}$ //ftp.ibge.gov.br/Estimativas_de_Populaca o/Estimativas_2016/estimativa _2016_TCU.pdf $>$. Acesso em 14/11/2016.

$\begin{array}{lrr}\text { INSTITUTO } & \text { NACIONAL } & \text { DE } \\ \text { METEOROLOGIA. } & \text { Dados } & \text { do }\end{array}$ Monitoramento da estação Automática de Ariquemes/RO. Comunicação pessoal via email: <sadmet.df@inmet.gov.br> em 18 de jun. 2014.
LACERDA, Z. C. ; TURCO, J. E. P. Estimation methods of reference evapotranspiration (ETo) for Uberlândia -MG. Engenharia Agrícola., v.35, n.1, p.27-38, 2015.

MAGALHÃES, F. F.; CUNHA, F. F. Desempenho do software SEVAP na estimativa da evapotranspiração no Estado de Mato Grosso do Sul. Revista Agrária, v.5, n.16, p.151-160, 2012.

MENDONÇA, J. C.; SOUSA, E. F.; BERNARDO, S.; DIAS, G. P.; GRIPPA, S. Comparação entre métodos de estimativa da evapotranspiração de referência (ETo) na região Norte Fluminense, RJ. Revista Brasileira de Engenharia Agrícola e Ambiental, v.7, n.2, p.275-279, 2003.

MENDOZA, C. J.; MENEZES, R. H., DIAS, A. S. Estimativa da evapotranspiração de referência por diferentes métodos para o município de São Luís - MA. Revista Brasileira de Agricultura Irrigada, v.10, n.3, p. 621 - 630, 2016.

MONTGOMERY, D. C.; RUNGER, G. C. Estatística aplicada e probabilidade para engenheiros. Tradução: Verônica Calado. 4a . Edição - Rio de Janeiro: LTC, 2009.

OLIVEIRA, M. A. A.; CARVALHO, D. F. de Estimativa da evapotranspiração de referência e da demanda suplementar de irrigação para o milho (Zeamays L.) em Seropédica e Campos, estado do Rio de Janeiro. Revista Brasileira de Engenharia Agrícola e Ambiental, v.2, n.2, p.132-135, 1998.

OLIVEIRA, R. Z.; OLIVEIRA, L. F. C.; WEHR, T. R.; BORGES, L. B.; BONOMO, R. Comparação de metodologias de estimativa da evapotranspiração de referência para a região de Goiânia, GO. Biosciense Journal, v.21, n.3, p.19-27, 2005.

PALARETTI, L. F.; MANTOVANI, E. C.; SEDIYAMA, G. C. Análise da sensibilidade dos componentes da equação de Hargreaves Samani para a região de Bebedouro - SP. 
Revista Brasileira de Meteorologia, v.29, n.2, p.299-306, 2014.

PEIXOTO, T. D. C.; LEVIEN, S. L. A.; BEZERRA, A. H. F.; SOBRINHO, J. E. Evapotranspiração de referência utilizando métodos de tanque classe $\mathrm{A}$ propostos pela FAO, na região de Mossoró, RN. Enciclopédia biosfera, v.6, n.11, p.1-7, 2010.

PEREIRA, D. R.; YANAGI, S. N. M.; MELLO, C. R.; SILVA, A. M.; SILVA, L. A.; Desempenho de métodos de estimativa da evapotranspiração de referência para a região da Serra da Mantiqueira, MG. Ciência Rural, v.39, n.9, p.2488-2493, 2009.

SANCHES, F. M.; XIMENES, A. R.; CORADI, P. C.; ROQUE, C. G.; CUNHA, F. F. Estimativa da evapotranspiração de referência na região norte do Brasil. Revista de Ciências Agroambientais, v.13, n.2, p.19-31, 2015.
SCALOPPI, E. J.; GARCIA, C. J. B. Estimativa simplificada da evapotranspiração de referência. Revista Brasileira de Agricultura Irrigada, v.9, n.6, p.376-382, 2015.

TAGLIAFERRE, C.; SILVA, J. P.; PAULA, A.; GUIMARAES, D. U. G.; BARROSO, N. I. S. Estimativa da evapotranspiração de referência para três localidades do estado da Bahia. Revista Caatinga, v.25, n.2, p.136-143, 2012.

TESTEZLAF, R. Irrigação: Métodos, Sistemas e Aplicações. Ed. não revisada. Campinas, SP: Faculdade de Engenharia Agrícola/UNICAMP 2011. 203p.

WILLMOTT, C. J.; AKLESON, G. S.; DAVIS, R. E.; FEDDEMA, J. J.; KLINK, K. M.; LEGATES, D. R.; ODONNELL, J.; ROWE, C. M. Statistic for the evaluation and comparison of models. Journal of Geophysical Research, v.90, p.8995-9005, 1985. 